\title{
Belumosudil: First Approval
}

\author{
Hannah A. Blair ${ }^{1}$ \\ Published online: 31 August 2021 \\ (c) Springer Nature 2021, corrected publication 2021
}

\begin{abstract}
Belumosudil (REZUROCK ${ }^{\mathrm{TM}}$ ) is a Rho-associated coiled-coil-containing protein kinase (ROCK) inhibitor that has been developed by Kadmon Pharmaceuticals for the treatment of chronic graft-versus-host disease (cGVHD) and systemic sclerosis. In July 2021, belumosudil received its first approval in the USA for the treatment of adult and paediatric patients aged $\geq 12$ years with cGVHD after failure of at least two prior lines of systemic therapy. Belumosudil is under regulatory review in Australia, Canada, the UK and Switzerland for cGVHD. Clinical development for systemic sclerosis is ongoing in the USA. This article summarizes the milestones in the development of belumosudil leading to this first approval for the treatment of cGVHD.
\end{abstract}

Digital Features for this AdisInsight Report can be found at https://
doi.org/10.6084/m9.figshare.15183840.

\section{Belumosudil (REZUROCK'm): Key Points}

ROCK inhibitor being developed by Kadmon Pharmaceuticals for the treatment of cGVHD and systemic sclerosis

Received its first approval on 16 July 2021 in the USA

Approved for the treatment of adult and paediatric patients aged $\geq 12$ years with $c G$ VHD after failure of at least two prior lines of systemic therapy

\section{Introduction}

Belumosudil (REZUROCK ${ }^{\mathrm{TM}}$ ) is an orally active rhoassociated coiled-coil-containing protein kinase (ROCK) inhibitor developed by Kadmon Pharmaceuticals for the

This profile has been extracted and modified from the AdisInsight database. AdisInsight tracks drug development worldwide through the entire development process, from discovery, through preclinical and clinical studies to market launch and beyond.

Hannah A. Blair

dru@adis.com

1 Springer Nature, Mairangi Bay, Private Bag 65901, Auckland 0754, New Zealand treatment of chronic graft-versus-host disease (cGVHD) and systemic sclerosis. Under its Real-Time Oncology Review pilot program, the US FDA granted belumosudil orphan drug [1] and breakthrough therapy [2] designations for the treatment of cGVHD. In November 2020, the US FDA accepted the New Drug Application and granted a priority review [3]. On 16 July 2021, 6 weeks ahead of the PDUFA goal date, belumosudil received its first approval in the USA for the treatment of adult and paediatric patients aged $\geq 12$ years with cGVHD after failure of at least two prior lines of systemic therapy [4, 5]. The recommended dosage of belumosudil is $200 \mathrm{mg}$ once daily (with food) until progression of cGVHD that requires new systemic therapy [4]. The dosage of belumosudil should be increased to $200 \mathrm{mg}$ twice daily in patients receiving concomitant strong CYP3A4 inducers or proton pump inhibitors [4].

Belumosudil is under regulatory review in Australia, Canada, the UK and Switzerland for CGVHD, and is in phase II development for cGVHD in China. Phase II development is ongoing in the USA for the treatment of systemic sclerosis. Development of belumosudil for the treatment of idiopathic pulmonary fibrosis, plaque psoriasis, non-alcoholic steatohepatitis, rheumatoid arthritis and systemic lupus erythematosus has been discontinued.

\subsection{Company Agreements}

In April 2011, Kadmon Pharmaceuticals entered into an agreement with Nano Terra under which Kadmon 


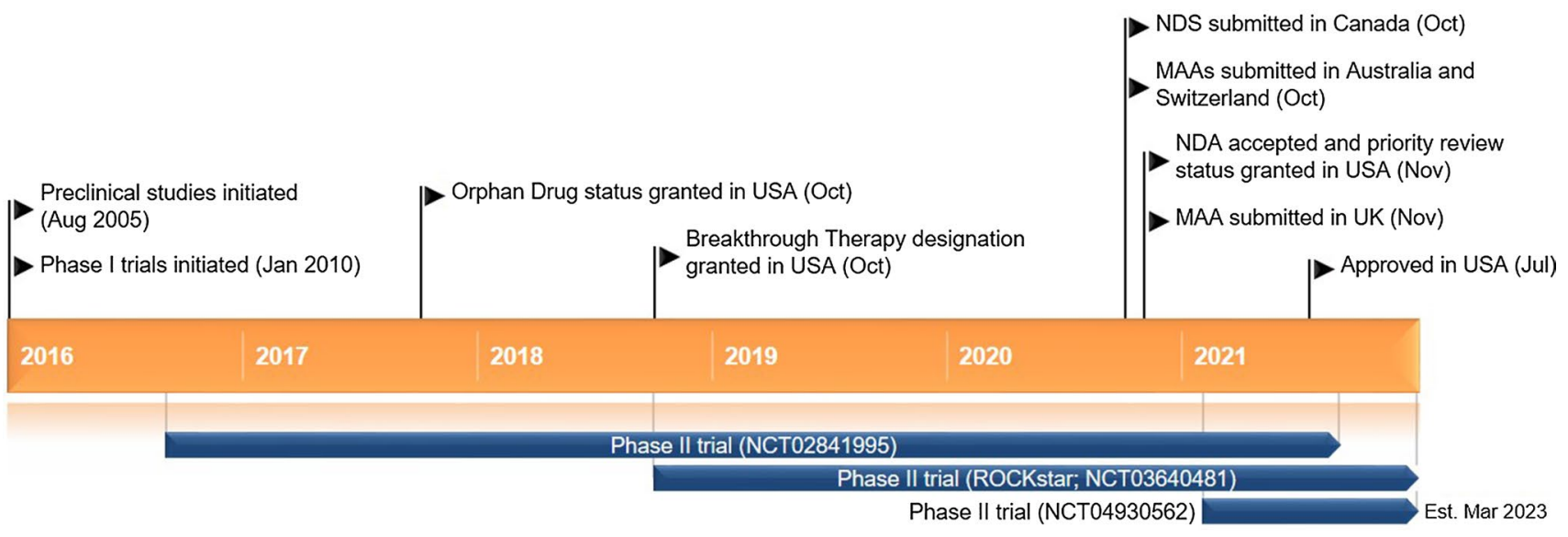

Key milestones in the development of belumosudil, focusing on its use in the treatment of chronic graft-versus-host disease. MAA Marketing Authorisation Application, NDA New Drug Application, NDS New Drug Submission

Pharmaceuticals was granted a perpetual, worldwide exclusive license to three of Nano Terra's novel product candidates (including belumosudil), as well as rights to its drug discovery platform Pharmacomer ${ }^{\mathrm{TM}}$ Technology [6]. In November 2019, Kadmon Holdings and BioNova Pharmaceuticals formed a joint venture, BK Pharmaceuticals, to help accelerate the development and regulatory approval of belumosudil in China [7]. In December 2019, Kadmon Holdings and Meiji Seika Pharma formed a joint venture, Romeck Pharma, to exclusively develop and commercialise belumosudil in Japan and some other Asian countries [8].

\subsection{Patent Information}

As of December 2020, belumosudil is covered under a US patent with market exclusivity in the USA and Japan until 2033. Kadmon Pharmaceuticals also has composition of matter and method of use patent protection for belumosudil with market exclusivity in the USA, Canada, China, Japan and Eurasia until 2026-2029. The company also has pending patent applications for belumosudil in the USA, Canada, China, Japan, Eurasia and Europe covering method of use with market exclusivity until 2033-2035.

\section{Scientific Summary}

\subsection{Pharmacodynamics}

Belumosudil is a ROCK inhibitor with half-maximal inhibitory concentrations $\left(\mathrm{IC}_{50} \mathrm{~s}\right)$ of $\approx 100 \mathrm{nM}$ and $3 \mu \mathrm{M}$ for ROCK2 and ROCK1, respectively [4]. Belumosudil downregulates proinflammatory responses by inhibiting signal transducer and activator of transcription 3 (STAT3) phosphorylation, upregulating STAT5 phosphorylation and shifting T helper 17 (Th17)/T regulatory (Treg) balance towards the Treg phenotype $[9,10]$.

Belumosudil effectively ameliorated cGVHD in multiple murine models [9]. In vitro, belumosudil inhibited aberrant pro-fibrotic signalling [4] via downregulation of pro-fibrotic gene transcription, stress fibre formation, myofibroblast activation and collagen deposition [11]. In human<smiles>CC(C)NC(=O)COc1cccc(-c2nc(Nc3ccc4[nH]ncc4c3)c3ccccc3n2)c1</smiles>

Chemical structure of belumosudil 
fibrosis-derived smooth muscle cells, belumosudil reduced connective tissue growth factor mRNA and remodelled the actin cytoskeleton [12]. In the lung endothelium, belumosudil promoted oxidative phosphorylation, inhibited glycolysis, intracellular $\mathrm{pH}$ and migration, and strengthened barrier integrity [13].

\subsection{Pharmacokinetics}

Approximately dose-proportional increases in belumosudil maximum plasma concentration $\left(\mathrm{C}_{\max }\right)$ and area under the plasma concentration-time curve (AUC) are seen over a dosage range of 200-400 mg once daily [4]. The accumulation ratio of belumosudil is 1.4. Following once- or twicedaily administration of belumosudil $200 \mathrm{mg}$ in patients with cGVHD, the median time to $\mathrm{C}_{\max }$ at steady state was $1.26-2.53 \mathrm{~h}$. When a single dose of belumosudil was administered with a high-fat and high-calorie meal, belumosudil $\mathrm{C}_{\max }$ and AUC decreased 2.2-fold and 2-fold, respectively, while the median time to $\mathrm{C}_{\max }$ was extended by $0.5 \mathrm{~h}$. Following a single dose, belumosudil has a mean bioavailability of $64 \%$ [4].

The geometric mean volume of distribution of belumosudil is $184 \mathrm{~L}$ following a single dose [4]. The drug is $99.9 \%$ bound to human serum albumin and $98.6 \%$ bound to human $\alpha_{1}$-acid glycoprotein. Belumosudil metabolism is mediated primarily by CYP3A4 and, to a lesser extent, by CYP2C8, CYP2D6 and UGT1A9. Following a single oral dose of radiolabeled belumosudil, $85 \%$ of the radioactivity is excreted in the faeces ( $30 \%$ as unchanged drug) and $<5 \%$ is excreted in the urine. In patients with cGVHD, the mean elimination half-life of belumosudil is $19 \mathrm{~h}$ and the clearance is $9.83 \mathrm{~L} / \mathrm{h}$. Age (18-77 years), sex, bodyweight (38.6-143 kg) and mild to moderate renal impairment [estimated glomerular filtration rate (eGFR) $\geq 60$ and $<90 \mathrm{~mL} / \mathrm{min} / 1.73 \mathrm{~m}^{2}$ to eGFR $\geq 30$ and $<60 \mathrm{~mL} /$ $\mathrm{min} / 1.73 \mathrm{~m}^{2}$ ] had no clinically meaningful effect on the pharmacokinetics of belumosudil [4].

Belumosudil is an inhibitor of CYP1A2, CYP2C19, CYP2D6, UGT1A1 and UGT1A9, and also inhibits P-glycoprotein, BCRP and OATP1B1 at clinically relevant concentrations [4]. Belumosudil is a substrate of $\mathrm{P}$ glycoprotein. Coadministration of belumosudil with strong CYP3A inducers (e.g. rifampin) or proton pump inhibitors (e.g. rabeprazole, omeprazole) decreases the $\mathrm{C}_{\max }$ and AUC of belumosudil, which may reduce its efficacy. Coadministration of belumosudil with midazolam (a CYP3A substrate) is predicted to increase the $\mathrm{C}_{\max }$ and AUC of midazolam $\approx 1.3$ - and 1.5 -fold, respectively [4].

\section{Features and properties of belumosudil}

\begin{tabular}{|c|c|}
\hline Alternative names & Belumosudil mesylate; KD-025; KD025 mesylate; BN-101; SLX-2119; REZUROCK ${ }^{\mathrm{TM}}$ \\
\hline Class & $\begin{array}{l}\text { Acetamides; amines; anti-inflammatories; antifibrotics; antipsoriatics; antirheumatics; hepatoprotectants; } \\
\text { indazoles; phenyl ethers; quinazolines; skin disorder therapies; small molecules }\end{array}$ \\
\hline Mechanism of action & ROCK inhibitor \\
\hline Route of administration & Oral \\
\hline Pharmacodynamics & $\begin{array}{l}\text { Downregulates proinflammatory responses by inhibiting STAT3 phosphorylation, upregulating STAT5 phos- } \\
\text { phorylation and shifting Th17/Treg balance towards Treg phenotype }\end{array}$ \\
\hline Pharmacokinetics & $\begin{array}{l}\text { Median time to } \mathrm{C}_{\max } \text { at steady state } 1.26-2.53 \mathrm{~h} \text {; mean bioavailability } 64 \% \text {; geometric mean volume of distri- } \\
\text { bution } 184 \mathrm{~L} \text {; mean elimination half-life } 19 \mathrm{~h} \text {; clearance } 9.83 \mathrm{~L} / \mathrm{h}\end{array}$ \\
\hline Most frequent AEs & $\begin{array}{l}\text { Infections, asthenia, nausea, diarrhoea, dyspnoea, cough, oedema, haemorrhage, abdominal pain, musculo- } \\
\text { skeletal pain, headache, } \downarrow \text { phosphate, } \uparrow \text { GGT, } \downarrow \text { lymphocytes, hypertension }\end{array}$ \\
\hline \multicolumn{2}{|l|}{ ATC codes } \\
\hline WHO ATC code & L04A-A48 (belumosudil) \\
\hline EphMRA ATC code & L4 (immunosuppressants) \\
\hline Chemical name & 2-[3-[4-(1H-indazol-5-ylamino)quinazolin-2-yl]phenoxy]- $N$-propan-2-ylacetamide \\
\hline
\end{tabular}

$\downarrow$ decreased, $\uparrow$ increased, $A E s$ adverse events, $C_{\max }$ maximum plasma concentration, $G G T \gamma$-glutamyl transferase, $R O C K$ rho-associated coiledcoil-containing protein kinase, STAT signal transducer and activator of transcription, Th17 T helper 17 cells, Treg regulatory T cells 


\subsection{Therapeutic Trials}

\subsubsection{Chronic Graft-Versus-Host Disease}

The efficacy of belumosudil for the treatment of cGVHD was demonstrated in the randomized, multicentre, phase II ROCKstar trial in allogeneic haematopoietic cell transplant (alloHCT) recipients (NCT03640481) [14]. After a median follow-up of 14 months, the best overall response rate (ORR, defined as complete response or partial response; primary endpoint) was $74 \%$ (95\% CI 62-84\%) with belumosudil $200 \mathrm{mg}$ once daily and $77 \%$ (95\% CI 65-87\%) with belumosudil $200 \mathrm{mg}$ twice daily. Responses were observed in all affected organ systems and across all patient subgroups. The ORR was $74 \%$ in patients who previously received ibrutinib $(n=46)$ and $68 \%$ in patients who previously received ruxolitinib $(n=38)$. In this study, 132 patients aged $\geq 12$ years with persistent cGVHD manifestations after receiving $2-5$ prior systemic lines of therapy were randomized to receive oral belumosudil $200 \mathrm{mg}$ once or twice daily until clinically significant progression of cGVHD or unacceptable toxicity. Randomization was stratified by cGVHD severity and prior exposure to ibrutinib. At baseline, $99 \%$ of patients were receiving concomitant corticosteroids. The median time to response was 5 weeks, with most (91\%) responses seen within the first 6 months of treatment. Responses were maintained for $\geq 20$ weeks in $59 \%$ of patients. The median duration of response was 54 weeks. Clinically meaningful improvement ( $\geq 7$-point reduction) from baseline in the Lee Symptom Scale (LSS) score was observed in 59\% of patients receiving belumosudil $200 \mathrm{mg}$ once daily and $62 \%$ of patients receiving belumosudil $200 \mathrm{mg}$ twice daily; LSS improvements were seen in both responders and nonresponders. Corticosteroid doses were reduced in $65 \%$ of patients. The overall failure-free survival (FFS) rate was $75 \%$ at 6 months and $56 \%$ at 12 months. The overall survival (OS) rate at 2 years was $89 \%$ [14].

In an earlier open-label, multicentre, phase II, dose-finding trial in allogeneic bone marrow transplant or alloHCT recipients (NCT02841995), belumosudil was an effective treatment for cGVHD [15]. After a median follow-up of 29 months, 65\% (95\% CI 51-77\%) had an ORR (primary endpoint). Responses were seen in all affected organs and across all patient subgroups, including patients who had received $\geq 2$ prior systemic lines of therapy and patients with $\geq 4$ organs involved. The study enrolled 54 patients aged $\geq 18$ years with persistent cGVHD manifestations after 1-3 prior systemic lines of therapy and who were receiving corticosteroids with or without a calcineurin inhibitor and/or concurrent extracorporeal photopheresis. Patients in three sequential cohorts received oral belumosudil $200 \mathrm{mg}$ once daily, $200 \mathrm{mg}$ twice daily or $400 \mathrm{mg}$ once daily in 28-day cycles until cGHVD progression or unacceptable toxicity. Most (>75\%) responses were achieved during the first 8 weeks of treatment and the median duration of response was 35 weeks. Fifty percent of patients reported a clinically meaningful improvement in LSS score and $67 \%$ of patients were able to reduce their corticosteroid dose. The median time to next treatment was 14 months. FFS rates at 6,12 and 24 months were 76,47 and $33 \%$, respectively, and the 2-year OS rate was $82 \%$ [15].

\begin{tabular}{|c|c|c|c|c|c|c|}
\hline \multicolumn{7}{|c|}{ Key clinical trials of belumosudil } \\
\hline Drug & Indication & Phase & Status & Location & Sponsor & Identifier \\
\hline Belumosudil & cGVHD & II & Recruiting & USA & $\begin{array}{l}\text { Kadmon } \\
\text { Corporation }\end{array}$ & ROCKstar; NCT03640481 \\
\hline Belumosudil & cGVHD & II & $\begin{array}{l}\text { Active, no longer } \\
\text { recruiting }\end{array}$ & USA & $\begin{array}{l}\text { Kadmon } \\
\text { Corporation }\end{array}$ & NCT02841995 \\
\hline Belumosudil & cGVHD & II & Recruiting & China & $\begin{array}{l}\text { BioNova } \\
\text { Pharmaceuticals }\end{array}$ & NCT04930562 \\
\hline Belumosudil & $\begin{array}{l}\text { Systemic } \\
\text { sclerosis }\end{array}$ & II & Recruiting & USA & $\begin{array}{l}\text { Kadmon } \\
\text { Corporation }\end{array}$ & NCT04680975 \\
\hline Belumosudil & $\begin{array}{l}\text { Systemic } \\
\text { sclerosis }\end{array}$ & II & Recruiting & USA & $\begin{array}{l}\text { Kadmon } \\
\text { Corporation }\end{array}$ & NCT03919799 \\
\hline
\end{tabular}

$c G V H D$ chronic graft-versus-host disease 


\subsection{Adverse Events}

Belumosudil was well tolerated when used to treat cGVHD in phase II trials $[14,15]$. In a pooled analysis of both trials, the most common (incidence $\geq 20 \%$ ) AEs with belumosudil $200 \mathrm{mg}$ once daily $(n=83)$ were infection $(53 \%)$, asthenia $(46 \%)$, nausea (42\%), diarrhoea (35\%), dyspnoea (33\%), cough $(30 \%)$, oedema (27\%), haemorrhage (23\%), musculoskeletal pain (22\%), abdominal pain (22\%), headache $(21 \%)$ and hypertension (21\%) [4]. The most common (incidence $\geq 5 \%$ ) grade 3-4 AEs, including laboratory abnormalities, were infection (16\%), decreased lymphocytes (13\%), increased $\gamma$-glutamyl transferase (11\%), hypertension (7\%), decreased phosphate $(7 \%)$, decreased platelets $(5 \%)$, diarrhoea $(5 \%)$, dyspnoea (5\%) and haemorrhage (5\%). AEs leading to discontinuation of belumosudil occurred in $18 \%$ of patients while AEs leading to dose interruption occurred in $29 \%$ of patients. One patient experienced a fatal AE (severe nausea, vomiting, diarrhoea and multi-organ failure) [4].

Based on animal studies and its mechanism of action, belumosudil may cause foetal harm when administered to pregnant women, who should be advised of the potential risk [4]. Breastfeeding is not recommended during treatment with belumosudil and for $\geq 1$ week after the last dose due to the potential for belumosudil to be excreted in milk, which could lead to serious adverse reactions [4].

\subsection{Ongoing Clinical Trials}

In addition to the ongoing phase II cGVHD trials described above (NCT03640481 and NCT02841995), an open-label, multicentre, phase II trial (NCT04930562) is currently recruiting patients in China. The trial plans to evaluate the efficacy and safety of belumosudil $200 \mathrm{mg}$ once daily in patients with cGVHD after at least first line of systemic therapy.

Patients with diffuse cutaneous systemic sclerosis are being recruited in two phase II trials: an open-label, multicentre trial evaluating the efficacy and safety of belumosudil $200 \mathrm{mg}$ twice daily (NCT04680975) and a randomized, double-blind, placebo-controlled trial with an open-label extension evaluating the efficacy and safety of belumosudil $200 \mathrm{mg}$ once or twice daily (NCT03919799).

\section{Current Status}

Belumosudil received its first approval in the USA on 16 July 2021 for the treatment of adult and paediatric patients aged $\geq 12$ years with cGVHD after failure of at least two prior lines of systemic therapy [5].
Supplementary Information The online version contains supplementary material available at https://doi.org/10.1007/s40265-021-01593-z.

\section{Declarations}

Funding The preparation of this review was not supported by any external funding.

Authorship and Conflict of interest During the peer review process the manufacturer of the agent under review was offered an opportunity to comment on the article. Changes resulting from any comments received were made by the authors on the basis of scientific completeness and accuracy. Hannah Blair is a salaried employee of Adis International Ltd/Springer Nature, and declares no relevant conflicts of interest. All authors contributed to the review and are responsible for the article content.

Ethics approval, Consent to participate, Consent to publish, Availability of data and material, Code availability Not applicable.

Open Access This article is licensed under a Creative Commons Attribution-NonCommercial 4.0 International License, which permits any non-commercial use, sharing, adaptation, distribution and reproduction in any medium or format, as long as you give appropriate credit to the original author(s) and the source, provide a link to the Creative Commons licence, and indicate if changes were made. The images or other third party material in this article are included in the article's Creative Commons licence, unless indicated otherwise in a credit line to the material. If material is not included in the article's Creative Commons licence and your intended use is not permitted by statutory regulation or exceeds the permitted use, you will need to obtain permission directly from the copyright holder. To view a copy of this licence, visit http://creativecommons.org/licenses/by-nc/4.0/

\section{References}

1. Kadmon Holdings. FDA grants orphan drug designation to Kadmon's KD025 for the treatment of cGVHD [media release]. 6 Oct 2017. http://www.kadmon.com.

2. Kadmon Holdings. FDA grants breakthrough therapy designation to Kadmon's KD025 for chronic graft-versus-host disease [media release]. 17 Oct 2018. http://www.kadmon.com.

3. Kadmon Holdings. Kadmon announces FDA acceptance of NDA for belumosudil in patients with chronic graft-versus-host disease [media release]. 30 Nov 2020. http://www.kadmon.com.

4. US FDA. REZUROCK ${ }^{\mathrm{TM}}$ (belumosudil) tablets, for oral use: US prescribing information. 2021. https://www.accessdata.fda.gov. Accessed 18 Aug 2021.

5. Kadmon Pharmaceuticals. US FDA grants full approval of REZUROCK $^{\mathrm{TM}}$ (belumosudil) for the treatment of patients with chronic graft-versus-host disease (cGVHD) [media release]. 16 Jul 2021. https://investors.kadmon.com.

6. Kadmon Pharmaceuticals LLC, Nano Terra Inc. Kadmon Pharmaceuticals and Nano Terra announce worldwide exclusive licensing agreement for three novel product candidates [media release]. 25 Apr 2011. http://www.kadmon.com. 
7. Kadmon Holdings. Kadmon establishes strategic partnership with BioNova to develop and commercialize KD025 for the treatment of GVHD in China [media release]. 2019. http://www.kadmon. com.

8. Kadmon Holdings, Meiji Seika Pharma Co. Ltd. Kadmon and Meiji announce collaboration to develop and commercialize KD025 in Japan [media release]. 7 Nov 2019. http://www.kadmon.com.

9. Flynn R, Paz K, Du J, et al. Targeted Rho-associated kinase 2 inhibition suppresses murine and human chronic GVHD through a STAT3-dependent mechanism. Blood. 2016;127(17):2144-54.

10. Zanin-Zhorov A, Weiss JM, Nyuydzefe MS, et al. Selective oral ROCK2 inhibitor down-regulates IL-21 and IL-17 secretion in human T cells via STAT3-dependent mechanism. Proc Natl Acad Sci USA. 2014;111(47):16814-9.

11. Kadmon Pharmaceuticals. About belumosudil. 2021. https:// kadmon.com/clinical-pipeline/pipeline/about-kd025/. Accessed 18 Aug 2021.
12. Boerma M, Fu Q, Wang J, et al. Comparative gene expression profiling in three primary human cell lines after treatment with a novel inhibitor of Rho kinase or atorvastatin. Blood Coagul Fibrinolysis. 2008;19(7):709-18.

13. Lee JY, Stevens RP, Kash M, et al. KD025 shifts pulmonary endothelial cell bioenergetics and decreases baseline lung permeability. Am J Respir Cell Mol Biol. 2020;63(4):519-30.

14. Cutler CS, Lee SJ, Arai S, et al. Belumosudil for chronic graft-versus-host disease (cGVHD) after 2 or more prior lines of therapy: the ROCKstar study. Blood. 2021. https://doi.org/10.1182/blood. 2021012021.

15. Jagasia M, Lazaryan A, Bachier CR, et al. ROCK2 inhibition with belumosudil (KD025) for the treatment of chronic graft-versushost disease. J Clin Oncol. 2021;39(17):1888-98. 